\title{
Metal-Organic Honeycomb Nanomeshes with Tunable Cavity Size
}

\author{
U. Schlickum, ${ }^{*, \dagger}$ R. Decker, ${ }^{\dagger}$ F. Klappenberger, ${ }^{\dagger,+}$ G. Zoppellaro, ${ }^{\ddagger}$ S. Klyatskaya, ${ }^{\ddagger}$ \\ M. Ruben, ${ }^{*, \neq}$ I. Silanes, ${ }^{\S}$ A. Arnau, ${ }^{\S}$ K. Kern, ${ }^{\dagger, \|}$ H. Brune, ${ }^{\dagger}$ and J. V. Barth ${ }^{\star, \dagger, \perp,+}$
}

Institut de Physique des Nanostructures, Ecole Polytechnique Fédérale de Lausanne, CH-1015 Lausanne, Switzerland, Institut für Nanotechnologie, Forschungszentrum Karlsruhe, D-76021 Karlsruhe, Germany, Donostia International Physics Center (DIPC) and Departamento de Fisica de Materiales and Unidad de Fisica de Materiales, E- 20018 San Sebastian, Spain, Max-Planck-Institut für Festkörperforschung, D-70569 Stuttgart, Germany, PHAS-AMPEL, University of British Columbia, 2355 East Mall, Vancouver V6T 1Z4, Canada, and Physik Department E20, Technische Universität München, D-85748 Garching, Germany

Received September 26, 2007; Revised Manuscript Received October 31, 2007

\begin{abstract}
We present a systematic study of metal-organic honeycomb lattices assembled from simple ditopic molecular bricks and Co atoms on $\mathrm{Ag}(111)$. This approach enables us to fabricate size- and shape-controlled open nanomeshes with pore dimensions up to $5.7 \mathrm{~nm}$. The networks are thermally robust while extending over $\mu \mathrm{m}^{2}$ large areas as single domains. They are shape resistant in the presence of further deposited materials and represent templates to organize guest species and realize molecular rotary systems.
\end{abstract}

Supramolecular chemistry with its unique control of highly organized molecular architecture and intrinsic defect correction is an efficient synthetic tool for nanoscale control of matter. In particular, the development of metallosupramolecular self-assembly techniques gives access to a variety of grid structures based on the coordination of organic linkers with metal centers. ${ }^{1-3}$ Recent studies revealed that lowdimensional coordination networks bearing potential for technological applications can be similarly realized on welldefined surfaces. ${ }^{4-6}$ Notably rectangular networks could be realized on square substrates featuring open cavities in the $0.5-2 \mathrm{~nm}$ range. ${ }^{5}$ Here, we report on a methodology to design highly regular size- and shape-controlled nanomeshes with tunable pore size, based on the transition metal-directed assembly of ditopic organic linkers on a $\mathrm{Ag}(111)$ substrate. The underlying threefold Co-carbonitrile coordination motif is examined with the help of first-principles calculations. By varying the length of the custom-designed polyphenyldicarbonitrile linkers, we realized open honeycomb networks comprising hexagonal pores up to $5.7 \mathrm{~nm}$ in diameter. The

* Corresponding authors. E-mail: (U.S.) uta.schlickum@epfl.ch; (M.R.) mario.ruben@int.fzk.de; (J.V.B.) jvb@ph.tum.de.

†cole Polytechnique Fédérale de Lausanne.

$\doteqdot$ Forschungszentrum Karlsruhe.

$\S$ Donostia International Physics Center (DIPC) and Departamento de Fisica de Materiales and Unidad de Fisica de Materiales.

"Max-Planck-Institut für Festkörperforschung.

$\perp$ University of British Columbia.

+ Technische Universität München. pertaining shape-resistant nanocavities provide an ideal playground for many further studies. In particular, we demonstrate their capability to spatially confine guest species and observe their restricted lateral motion.

The objective of our investigation is the development of a rationale for surface-confined supramolecular chemistry, ${ }^{7}$ which will offer an exquisite tool for the massively parallel construction of extended, defect-free regular two-dimensional (2D) nanoporous networks..$^{5,8-11} \mathrm{We}$ devised a series of simple ditopic dicarbonitrile-polyphenyl molecular linkers (abbreviated $\mathrm{NC}-\mathrm{Ph}_{n}-\mathrm{CN}$, whereby $n$ can be 3 , 4, or 5; see Figure 1). A straightforward synthesis of the rodlike NC$\mathrm{Ph}_{n}-\mathrm{CN}$ molecules was developed on the basis of Suzuki reaction schemes, by the coupling of corresponding bisiodophenylens and two equivalents of 4-carbonitrile-phenylboronic acid under typical Suzuki conditions ${ }^{12}$ with 10 mol \% $\mathrm{Pd}(0)$. Corresponding bis-iodophenylens were achieved by iodination of polyphenylens with a mixture of $\mathrm{I}_{2}$ and $\mathrm{HIO}_{3}$ in acetic acid. All linkers gave correct analytical data and agreed with literature data for $\mathrm{NC}-\mathrm{Ph}_{3}-\mathrm{CN},{ }^{13} 4,4^{\prime}$-bisiododiphenyl, ${ }^{14}$ and $4,4^{\prime \prime}$-bisiodo-[1, $\left.1^{\prime} ; 4^{\prime}, 1^{\prime \prime}\right]$-terphenyl. ${ }^{15}$ After all organic compounds were purified by normal chemical methods, they were sublimed twice to obtain the purity needed for scanning tunneling microscopy (STM) experiments, performed in a home-built ultrahigh vacuum (base pressure $10^{-10}$ mbar) low-temperature STM setup. ${ }^{16}$ The $\mathrm{Ag}(111)$ substrate was prepared by cycles of $\mathrm{Ar}^{+}$sputtering 

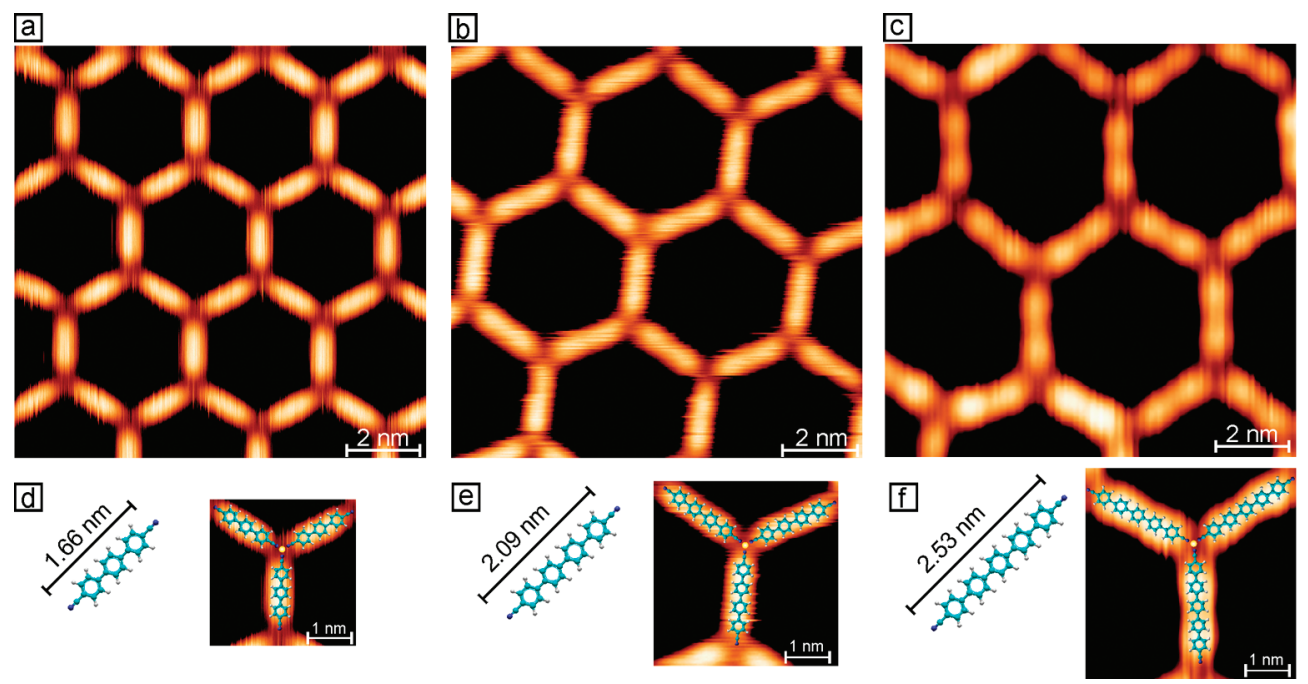

Figure 1. Tuning the cavity size of metal-organic honeycomb networks with designed linkers. The STM images show the result of Co-directed assembly of (a) $\mathrm{NC}-\mathrm{Ph}_{3}-\mathrm{CN}$, (b) $\mathrm{NC}-\mathrm{Ph}_{4}-\mathrm{CN}$, and (c) $\mathrm{NC}-\mathrm{Ph}_{5}-\mathrm{CN}$, respectively. (d-f) Structure of the molecules including their length and models of the threefold Co-carbonitrile coordination motif resolved in $(\mathrm{a}-\mathrm{c})$ (yellow, cobalt center; turquoise, carbon; white, hydrogen; blue, nitrogen). The images $(\mathrm{a}-\mathrm{c})$ were taken at a tunnel current of $I=0.1 \mathrm{nA}$ and bias voltages of $0.9,1$, and $2 \mathrm{~V}$, respectively.

and annealing to $800 \mathrm{~K}$ to obtain flat terraces separated by monatomic steps. The molecules were evaporated with organic molecular beam epitaxy at $460 \mathrm{~K}\left(\mathrm{NC}-\mathrm{Ph}_{3}-\mathrm{CN}\right)$, $490 \mathrm{~K}\left(\mathrm{NC}-\mathrm{Ph}_{4}-\mathrm{CN}\right)$, and $535 \mathrm{~K}\left(\mathrm{NC}-\mathrm{Ph}_{5}-\mathrm{CN}\right)$, respectively, onto a clean $\operatorname{Ag}(111)$ surface. We first deposited submonolayer amounts of the organic linkers (the corresponding pure organic networks formed by the respective molecular building blocks are rather complex and will be discussed in detail elsewhere). Subsequently, the precursor layers were exposed to a beam of Co atoms to fabricate the metal-organic networks. During all steps, the $\operatorname{Ag}(111)$ crystal was kept at $300 \mathrm{~K}$. By carefully adjusting the stoichiometry of the constituents, we achieved the presence of single metal-organic phases on the surface where all transition metal centers are incorporated (in the case of $\mathrm{Co}$, deficiency coexistence of nanomeshes and organic islands occurs; also an overdose of Co atoms is not affecting the metal-organic nanomesh because surplus Co atoms are accumulated in small clusters outside the network, decorating $\mathrm{Ag}$ step edges). After the preparation, the sample was transferred into the low-temperature STM where all data were recorded at $T \approx 10 \mathrm{~K}$ in the constant current mode.

All ditopic molecular bricks have the same functional carbonitrile endgroups, while their lengths increase with $n$ from 1.66 via 2.09 up to $2.53 \mathrm{~nm}$. Because carbonitrile compounds are known to coordinate strongly to transition metal centers, ${ }^{17,18}$ they represent promising candidates for the improved control of surface-confined metal-organic superlattices. Indeed, the STM data reproduced in Figure 1 demonstrates that by the controlled reaction of cobalt centers with preadsorbed linker molecules a series of open nanomeshes with a tunable cavity size can be realized on the employed clean and smooth $\mathrm{Ag}(111)$ substrate. In all cases, highly regular arrays comprising nanometer-sized honeycomb cavities are formed. The area of the enclosed hexagons increases stepwise with the number of phenyl rings incorporated in the molecular linkers' backbone. Accordingly, the cell size expands stepwise from $\approx 10$ via 15 up to $20 \mathrm{~nm}^{2}$ for $n=3,4,5$, respectively, (see Figure 1a-c). In particular, the $20 \mathrm{~nm}^{2}$ nanopores achieved with $\mathrm{NC}-\mathrm{Ph}_{5}-\mathrm{CN}$ linkers represent to the best of our knowledge the most open surfaceconfined nanomesh realized to date by 2D self-assembly.

All nanomeshes comprise the same threefold coordination motif with Co atoms positioned at the nodes (Figure 1d-f). The Co centers connect the molecules symmetrically resulting in an angle of $120^{\circ}$ between adjacent ligands. The domain size in known 2D nanoporous metal-organic networks was so far restricted to approximately $50 \times 50 \mathrm{~nm}^{2}$ (realized by $\mathrm{Fe}$ or Co carboxylation). In addition, an appreciable defect concentration within the domains was usually present, and controlling possibly coexisting phases can be very cumbersome. ${ }^{4,5,19,20}$ By exploiting Co-carbonitrile complexation, single domain metal-organic networks covering surface terraces over $\mu \mathrm{m}^{2}$ large areas with low defect concentrations become possible. This is illustrated by the data reproduced in Figure 2, where the regularity of the comprised hexagonal cavities is highlighted in the overview STM image (Figure 2a) and by a Fourier transformation taken from topographic data of a large scale image (Figure $2 b$ ) where distinct and sharp first and second-order peaks appear. Large domains are always obtained if the metal-organic network has been formed on the $\operatorname{Ag}(111)$ surface at a temperature of $300 \mathrm{~K}$ during the self-assembly process. The domains of the metalorganic arrangements are not restricted to single terraces because the network topology can be continued across atomic steps of the $\operatorname{Ag}(111)$ surface. The only known structure comparable in quality is the boron-nitride nanomesh. ${ }^{21} \mathrm{In}$ comparison to the latter, our metal-organic nanomeshes offer open cavities separated by molecular height boundaries. In addition, the cavity size is shown to be tunable and nearly triples the fabricated maximum mesh size with the NC$\mathrm{Ph}_{5}-\mathrm{CN}$ linkers.

The histogram in Figure 2c shows the size distribution of the hexagonal cells. The size given in the figure is the inner 

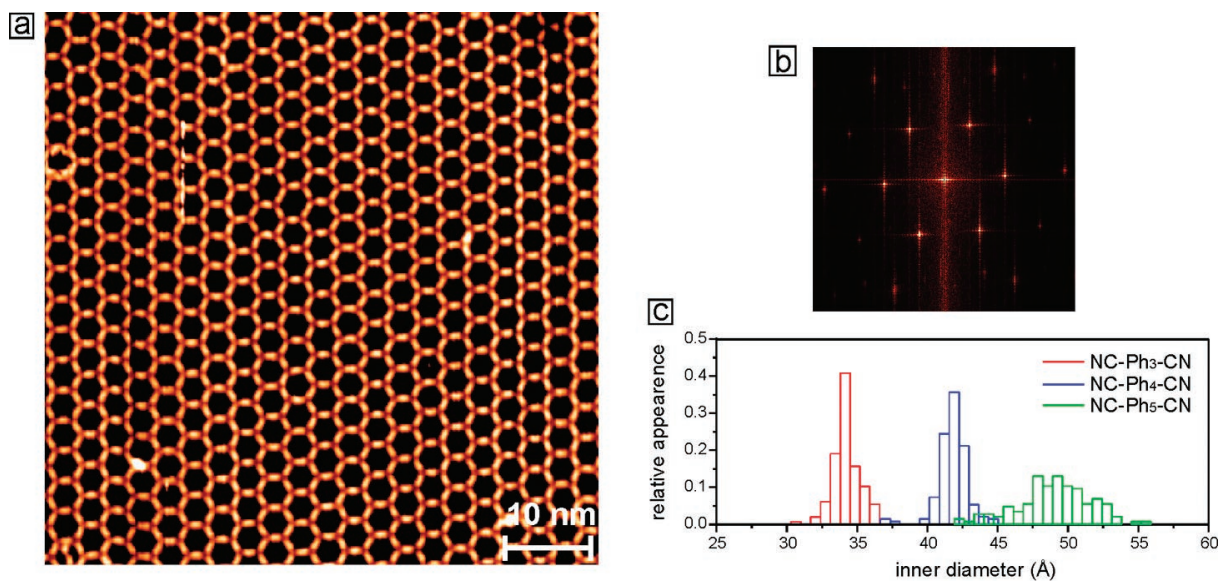

Figure 2. Extended highly regular nanomesh covering large areas. (a) Overview STM topographic image of the metal-organic network formed by $\mathrm{NC}-\mathrm{Ph}_{3}-\mathrm{CN}$ linkers. (b) Fourier transform taken from topographic data of a $300 \times 300 \mathrm{~nm}^{2}$ size domain with distinct first and second-order spots. (c) Histogram showing the size distribution of the cavities' inner diameter for $\mathrm{NC}^{-} \mathrm{Ph}_{3}-\mathrm{CN}-, \mathrm{NC}-\mathrm{Ph}_{4}-\mathrm{CN}-$, and $\mathrm{NC}-\mathrm{Ph}_{5}-\mathrm{CN}$-based networks.

diameter of the hexagons, that is, the distance between two parallel oriented molecules. The sharpness of the peaks for $\mathrm{NC}-\mathrm{Ph}_{3}-\mathrm{CN}$ and $\mathrm{NC}-\mathrm{Ph}_{4}-\mathrm{CN}$ (half width at halfmaximum (HWHM) for both are 3\%) underlines the nearly perfect geometric order within the metal-organic networks. The histograms have been established for large scale STM images, which broadens the distribution due to the limited pixel resolution. Therefore the real distribution can be assumed to be even narrower. The distribution for $\mathrm{NC}-\mathrm{Ph}_{5}-$ $\mathrm{CN}$ molecules is broader (HWHM is 7.8\%) indicating slight deviations from the threefold symmetry of the coordination node.

Density functional theory (DFT) calculations indicate that the encountered threefold coordination motif, uncommon for bulk transition metal-carbonitrile complexes, ${ }^{17,18}$ is induced by the presence of a metal substrate. ${ }^{22}$ For the modeling, the molecular linkers were simplified as $\mathrm{NC}-\mathrm{Ph}_{1}-\mathrm{CN}$ retaining the carbonitrile endgroups that interact with the $\mathrm{Co}$ centers. In addition, the molecules were confined in a plane in accordance with our data showing that the aromatic polyphenylene linkers are adsorbed in a flat configuration with the $\pi$-system facing the metal substrate. To assess the interaction of the Co center with the underlying surface, we performed calculations of both free planar compounds and complexes where a cluster of four $\mathrm{Ag}$ atoms was placed underneath the coordinated Co atom (Figure 3). We used the SIESTA code $^{23}$ with the Perdew-Burke-Ernzerhof functional, a double- $\zeta$ plus polarization basis set, a $100 \mathrm{meV}$ energy shift, and a 200 Ry cutoff for the spatial grid. The ligands were constrained to lie coplanar, whereas the $\mathrm{Co}$ atom was allowed to relax in any spatial direction. For the calculations including an $\mathrm{Ag}_{4}$ cluster under the $\mathrm{Co}$ atom, the distance between $\mathrm{Ag}$ atoms was kept frozen and the center of mass of the cluster was allowed to relax vertically (see Figure 3). The comparison between threefold and fourfold coordination was made by calculating the total binding energy of the complete system. The total binding energy is defined as the energy difference between the energy of the complete system and its constituent parts, consisting of the ligands, the Co atom, and the $\mathrm{Ag}_{4}$ cluster (if present). For
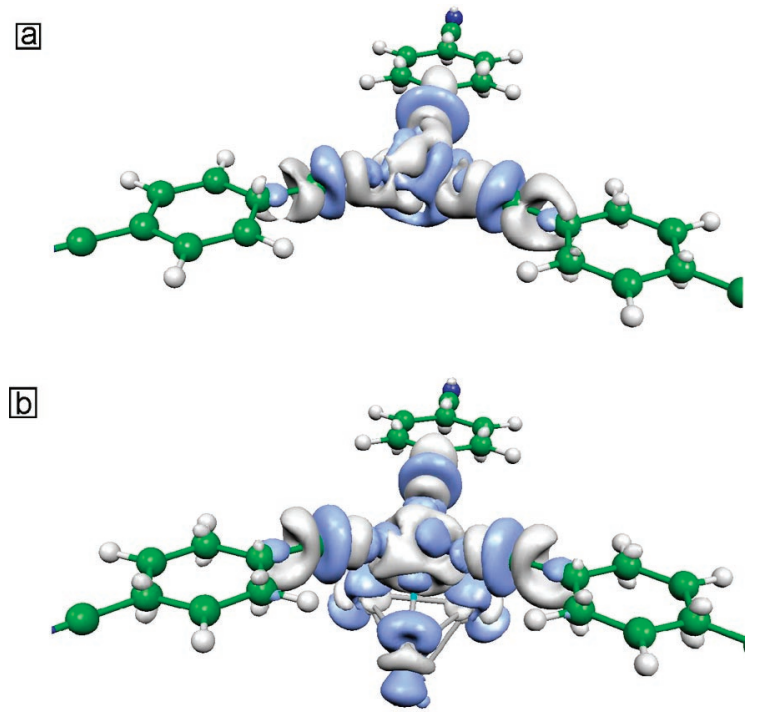

Figure 3. DFT calculations help to uncover the origin of threefold coordinated Co centers. (a,b) Plots of the induced charge density around the cobalt atom in threefold coordination of a model compound $\left(\mathrm{NC}-\mathrm{Ph}_{1}-\mathrm{CN}\right)$ without (a) and with (b) the presence of an $\mathrm{Ag}_{4}$ cluster underneath the transition metal center. The image displays the electron density redistribution around the Co atom due to the bond formation with the ligands and the $\mathrm{Ag}_{4}$ cluster. Light blue color means charge depletion, and light gray charge accumulation $\left(0.002 \mathrm{e} / \AA^{3}\right)$.

the case where no Ag cluster is placed below the Co center, the binding energy for fourfold coordination $(-5.46 \mathrm{eV})$ exceeds the that for threefold $(-5.92 \mathrm{eV})$ by $460 \mathrm{meV}$. When the $\mathrm{Ag}$ cluster is included, the energetic preference is reversed and now the binding energy for threefold coordination $(-7.88$ $\mathrm{eV})$ exceeds that for the fourfold $(-7.79 \mathrm{eV})$ by $90 \mathrm{meV}$.

The consideration of the binding energies within the node indicates that the interaction of the cobalt atoms with the surface is a key factor in favoring a $2 \mathrm{D}$ network with a threefold coordination of the organic ligands. The electron density redistributions in Figure 3 were calculated as the difference between the valence electron density of the total system and the sum of that of the two, three fragments, 
respectively (ligands and Co or ligands, $\mathrm{Co}$, and $\mathrm{Ag}_{4}$ ). The figure shows that the charge distribution between Co centers and carbonitrile ligands is not strongly affected when integrating the $\mathrm{Ag}_{4}$ cluster. On the other hand, the addition of the $\mathrm{Ag}_{4}$ cluster leads to a strong hybridization between $\mathrm{Co}$ and $\mathrm{Ag}$ atoms revealing the influence of the substrate in the coordination system. ${ }^{24}$ Qualitatively, the same behavior is observed for fourfold coordination; however, quantitative differences are encountered as apparent in the energy balance discussed above. This interpretation of a metal-metal bond influence on metal-ligand bonding is corroborated by a very similar coordination motif found for carbonitrile groups and the $\mathrm{Pd}(\mathrm{I})-\mathrm{Pd}(\mathrm{I})$ metallic complex favoring a threefold coordination $\left[\left(\mathrm{CH}_{3} \mathrm{CN}\right)_{3}-\mathrm{Pd}-\mathrm{Pd}-\left(\mathrm{CH}_{3} \mathrm{CN}\right)_{3}\right] .{ }^{25}$

Assuming that the molecular linkers have the same length on the surface as in the gas phase ${ }^{16}$ and that they are lying flat on the substrate (as indicated in Figure 1d-f), the projected $\mathrm{Co}-\mathrm{N}$ bond length obtained from high-resolution STM data is $(1.6 \pm 0.2) \AA$. This is consistent with our calculated value for threefold coordination and a Ag cluster considered below the coordination node and if we take into account the fact that the Co atom is out of the plane of the ligands $1.84 \AA$ ( $1.75 \AA$ projected). It also agrees well with other reported values for $\mathrm{Co}-\mathrm{N}$ bond length (cf. refs 18 and 26).

The metal-organic networks themselves provide a basis for a variety of further experiments because they are stable at temperatures up to $300 \mathrm{~K}$ and shape-persistent in the presence of additionally deposited materials, even for transition metal atoms $(\mathrm{Fe})$. The network stability was verified by repeated heating from 10 to $300 \mathrm{~K}$. After cooling down to $10 \mathrm{~K}$, the original honeycomb structure was always present and only an increase of impurities on top of the network could be discerned. Thus, the honeycomb networks can serve as templates to organize coadsorbed molecular species and to study molecular motion processes in confined environments. ${ }^{27-29}$ As a test case, we employed the hexagonal pores as host cavities for guest molecules. The template reproduced in Figure $4 \mathrm{a}$ is given by a network of $\left[\left(\mathrm{NC}-\mathrm{Ph}_{4}-\mathrm{CN}\right)_{3 / 2} \mathrm{Co}\right]$ complexes. We added $\mathrm{NC}-\mathrm{Ph}_{5}-\mathrm{CN}$ molecules to it at a substrate temperature of $\approx 150 \mathrm{~K}$. The longer guest molecules are accommodated in the cavities and arranged in specific configurations. By deliberate excitation with the STM tip, that is, increasing the tunnel current from 0.1 to $0.4 \mathrm{nA}$, the molecules can be laterally displaced from one configuration to another (see the two states depicted in Figure 4a,b). As another example, $\mathrm{NC}-\mathrm{Ph}_{3}-\mathrm{CN}$ linkers confined in the cavities formed by $\left[\left(\mathrm{NC}-\mathrm{Ph}_{3}-\mathrm{CN}\right)_{3 / 2} \mathrm{Co}\right]$ networks can be rotated back and forth between two metastable positions (Figure $4 \mathrm{c}-\mathrm{e}$ ). The achieved molecular organization and the tip-induced motions within the cavities reveal that networks qualify for spatial confinement of guest species.

In conclusion, we fabricated surface-supported highly regular metal-organic nanomeshes covering terraces over $\mu \mathrm{m}^{2}$ large areas as single domains by exploiting the coordinative interaction of carbonitrile groups in ditopic linear polyphenyl linkers with Co centers. By metallosupramolecular engineering, the mesh size of the formed honeycomb
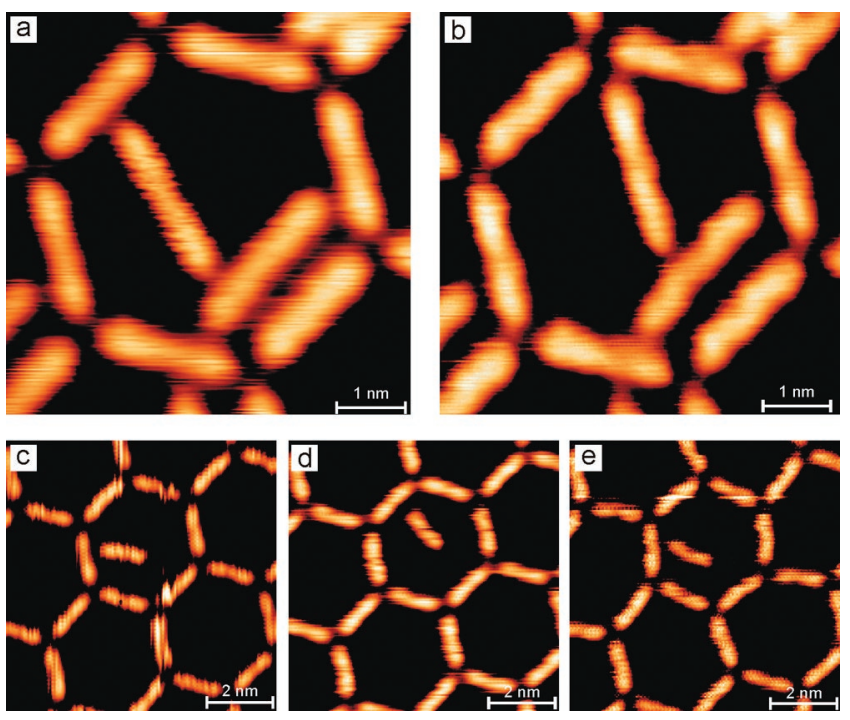

Figure 4. Nanocavities for confinement of molecular motion in a controlled environment. (a,b) A template formed by $\mathrm{NC}-\mathrm{Ph}_{4}-$ $\mathrm{CN}$ linkers is used to host two $\mathrm{NC}-\mathrm{Ph}_{5}-\mathrm{CN}$ guest molecules. We can laterally displace the $\mathrm{NC}-\mathrm{Ph}_{5}-\mathrm{CN}$ by increasing the tunnel current from 0.1 to $0.4 \mathrm{nA}$. (c-e) Host cavities, formed by the metal-organic network of $\mathrm{NC}-\mathrm{Ph}_{3}-\mathrm{CN}$ linkers where a single $\mathrm{NC}-\mathrm{Ph}_{3}-\mathrm{CN}$ guest is trapped. The confined species can be switched between two configurations by changing the scan direction (transition from configuration $\mathrm{c}$ to configuration d) or by increasing the tunnel current from 0.1 to $0.45 \mathrm{nA}$ (transition from configuration $\mathrm{d}$ to configuration e). Bias voltages: $1 \mathrm{~V}(\mathrm{a}-\mathrm{c})$ and $0.8 \mathrm{~V}(\mathrm{~d}, \mathrm{e})$, respectively.

arrays can be tailored under maintenance of their specific shape and symmetry, whereby they exhibit a high degree of regularity. The findings for $\mathrm{NC}-\mathrm{Ph}_{5}-\mathrm{CN}$ molecules show the most open 2D pore sizes designed by self-assembly processes on surfaces to date. Furthermore, the fabrication of size- and shape-controlled nanomeshes bears potential for a diversity of different applications. Notably, the capability of the metal-organic networks to show molecular motion in well-defined nanoscale environments was demonstrated. Studies of rotational processes of large nanometer size biomolecules in tunable spaces open ways to control molecular motors. Investigations of surface chemical reactions like catalytic processes in controlled surroundings are imaginable. Finally, the nanomeshes may serve as templates for the selective organization of guest species to realize patterned media of separated, regularly distributed nanoclusters.

Acknowledgment. Work supported by the European Science Foundation Collaborative Research Programme FunSMARTs. I.S. and A.A. thank UPV/EHU, Eusko Jaurlaritza and the Spanish MEC for partial financial support, and particularly the SGI/IZO-SGIker UPV/EHU for generous allocation of computational resources.

\section{References}

(1) Holiday, B. J.; Mirkin, C. A. Angew. Chem., Int. Ed. 2002, 40, 20222043.

(2) Yaghi, O. M.; O’Keeffe, M.; Ockwig, N. W.; Chae, H. K.; Eddaoudi, M.; Kim, J. Nature 2003, 423 (6941), 705-714.

(3) Ruben, M.; Rojo, J.; Romero-Salguero, F. J.; Uppadine, L. H.; Lehn, J.-M. Angew. Chem., Int. Ed. 2004, 43, 3644-3662. 
(4) Dmitriev, A.; Spillmann, H.; Lin, N.; Barth, J. V.; Kern, K. Angew. Chem., Int. Ed. 2003, 41, 2670-2673.

(5) Stepanow, S.; Lingenfelder, M.; Dmitriev, A.; Spillmann, H.; Delvigne, E.; Lin, N.; Deng, X.; Cai, C.; Barth, J. V.; Kern, K. Nature Mat. 2004, 3, 229-233.

(6) Barth, J. V.; Costantini, G.; Kern, K. Nature 2005, 437, 671-679.

(7) Barth, J. V. Annu. Rev. Phys. Chem. 2007, 58, 375-407.

(8) Theobald, J. A.; Oxtoby, N. S.; Phillips, M. A.; Champness, N. R.; Beton, P. H. Nature 2003, 424, 1029-1031.

(9) Spillmann, H.; Dmitriev, A.; Lin, N.; Messina, P.; Barth, J. V.; Kern, K. J. Am. Chem. Soc. 2003, 125, 10725-10728.

(10) Stöhr, M.; Wahl, M.; Galka, C. H.; Riehm, T.; Jung, T. A.; Gade, L. H. Angew. Chem., Int. Ed. 2005, 44, 7394-7398.

(11) Pawin, G.; Wong, K. L.; Kwon, K.-Y.; Bartels, L. Science 2006, 313, 961-962.

(12) Miyara, N.; Suzuki, A. Chem. Rev. 1995, 95, 2457-2483.

(13) Collonge, J.; Buendia, J.; Sabadie, J. Bull. Soc. Chem. Fr. 1967, 4370-4374.

(14) Merkushev, E. B.; Simakhina, N. D.; Koveshnikova, G. M. Synthesis 1980, 6, 486-487.

(15) Merkushev, E. B.; Yudina, N. D. Zh. Org. Khim. 1981, 17, $2598-$ 2601.

(16) Clair, S.; Pons, S.; Seitsonen, A. P.; Brune, H.; Kern, K.; Barth, J. V. J. Phys. Chem. B 2004, 108, 19392-19397.

(17) Fehlhammer, W. P.; Fritz, M. Chem. Rev. 1993, 93, 1243-1280.

(18) Przychodzen, P.; Korzeniak, T.; Podgajny, R.; Sieklucka, B. Coord. Chem. Rev. 2006, 250, 2234-2260.

(19) Lingenfelder, M.; Spillmann, H.; Dmitriev, A.; Stepanow, S.; Lin, N.; Barth, J. V.; Kern, K. Chem.-Eur. J. 2004, 10, 1913-1919.

(20) Clair, S.; Pons, S.; Fabris, S.; Baroni, S.; Brune, H.; Kern, K.; Barth, J. V. J. Phys. Chem. B 2006, 110, 5627.
(21) Corso, M.; Auwärter, W.; Muntwiler, M.; Tamai, A.; Greber, T.; Osterwalder, J. Science 2004, 303 (5655), 217-220; Berner, S.; Corso, M.; Widmer, R.; Groening, O.; Laskowski, R.; Blaha, P.; Schwarz, K.; Goriachko, A.; Over, H.; Gsell, S.; Schreck, M.; Sachdev, H.; Greber, T.; Osterwalder, J. Angew. Chem., Int. Ed. 2007, 46, 5115-5119.

(22) Stepanow, S.; Lin, N.; Payer, D.; Schlickum, U.; Klappenberger, F.; Zoppellaro, G.; Ruben, M.; Brune, H.; Barth, J. V.; Kern, K. Angew. Chem., Int. Ed. 2007, 46, 710-713.

(23) Soler, J. M.; Artacho, E.; Gale, J. D.; Garcia, A.; Junquera, J.; Ordejón, P.; Sánchez-Portal, D. J. Phys. C: Cond. Matt. 2002, 14, $2745-2780$.

(24) Seitsonen, A. P.; Lingenfelder, M.; Spillmann, H.; Dmitriev, A.; Stepanow, S.; Lin, N.; Kern, K.; Barth, J. V. J. Am. Chem. Soc. 2006 126, 5634-5635

(25) Murahashi, T.; Nagai, T.; Okuno, T.; Matsutani, T.; Kurosawa, H. Chem. Comm. 2000, 1689-1690.

(26) Kawamoto, T.; Asai, Y.; Abe, S. Phys. Rev. B 1999, 60, 1299012993.

(27) Gimzewski, J. K.; Joachim, C.; Schlittler, R. R.; Langlais, V.; Tang, H.; Johannsen, I. Science 1998, 281, 531-533.

(28) Lin, N.; Dmitriev, A.; Weckesser, J.; Barth, J. V.; Kern, K. Angew. Chem., Int. Ed. 2002, 41, 4779.

(29) Wintjes, N.; Bonifazi, D.; Cheng, F.; Kiebele, A.; Stöhr, M.; Jung, T.; Spillmann, H.; Diederich, F. Angew. Chem., Int. Ed. 2007, 46, 4089-4092.

NL072466M 